Research Article

\title{
Position Tracking Control of Robotic System Subject to Matched and Mismatched Disturbances
}

\author{
Xia Liu $\left(\mathbb{D},{ }^{1}\right.$ Dandan Liu, ${ }^{2}$ and Hao Sheng ${ }^{1}$ \\ ${ }^{1}$ School of Electrical Engineering and Electronic Information, Xihua University, Chengdu 610039, China \\ ${ }^{2}$ School of Information and Engineering, Sichuan Tourism College, Chengdu 610100, China \\ Correspondence should be addressed to Xia Liu; xliu_uestc@yahoo.com
}

Received 5 July 2020; Revised 4 September 2020; Accepted 11 September 2020; Published 23 September 2020

Academic Editor: Xingling Shao

Copyright ( $\odot 2020$ Xia Liu et al. This is an open access article distributed under the Creative Commons Attribution License, which permits unrestricted use, distribution, and reproduction in any medium, provided the original work is properly cited.

For nonlinear coupled robotic system subject to matched and mismatched disturbances, this paper designs an adaptive disturbance observer-based exponential sliding mode controller to achieve position tracking. Firstly, matched disturbance, mismatched disturbance, and the derivative of mismatched disturbance are defined as the lumped disturbance in robotic system. Secondly, a nonlinear disturbance observer is constructed to estimate the lumped disturbance, and an adaptive law is proposed to estimate the bound of the lumped disturbance. Finally, an exponential sliding mode controller is derived by combining the nonlinear disturbance observer and exponential convergence law. Stability and tracking performance of the robotic system is analyzed via Lyapunov function approach. Simulation results show that, with the proposed approach, both matched and mismatched disturbances in robotic system can be effectively depressed while achieving position tracking.

\section{Introduction}

Robotic system is a nonlinear system with strong coupling [1] and subject to various disturbances [2], e.g., external environmental disturbances, internal parameter uncertainties, measurement noises, joint friction, unknown load, and unmodeled dynamics [3-5]. Disturbances in a robotic system can degrade the position tracking performance and even undermine its stability. In general, the disturbances in a robotic system can be divided into two types: matched disturbances and mismatched disturbances [6]. Matched disturbances stand for the case where the disturbances indirectly affect the states of the robotic system through the control input; i.e., both the disturbances and control input are in the same channel. For instance, the disturbances in robotic systems were regarded as matched disturbances in $[7,8]$. In contrast, mismatched disturbances stand for the case where the disturbances directly affect the states of the robotic system without control input, i.e., the disturbances and control input are not in the same channel. For example, the disturbances in robotic systems were regarded as matched disturbances and mismatched disturbances in $[9,10]$.

In order to compensate matched disturbances in a robotic system, disturbance observer is an effective way [11]. In [12], a composite controller composed of a nonlinear disturbance observer and backstepping control law was derived to counteract the effect of exogenous disturbances in robotic system. In [13], for Port-Hamiltonian systems subject to matched disturbances, a dynamic state-feedback controller composited with a frequency observer was proposed to compensate the disturbance and applied to a 2-degree-offreedom (DOF) robotic manipulator. In [14], a nonlinear disturbance observer for a robotic exoskeleton was designed to compensate the disturbance torques caused by unknown input saturation, fuzzy approximation errors, viscous friction, gravity, and payloads. In [15], a robust control method based on filter and disturbance observer was presented for a two-link manipulator with flexible joints to suppress the disturbances. Nevertheless, the disturbance observers in the above literatures can only compensate matched disturbances but not mismatched disturbances. 
In practical applications, robotic system is not only subject to matched disturbances but also mismatched disturbances. Since mismatched disturbances and control input are not in the same channel, it is more challenging to design a controller which can compensate mismatched disturbances than matched disturbances [16]. For a linear system with mismatched disturbances, a memoryless-based and a memory-based integral sliding mode controllers based on disturbance observer were proposed, respectively, which were applied to the ball and beam system in [17]. However, in [17], only continuous linear system is considered. For a repetitive-control system with mismatched disturbances, a generalized extended state observer-based repetitive-control method was designed to improve the disturbance rejection performance in [18]. For an affine nonlinear system with mismatched disturbances and uncertainties, a nonlinear disturbance observer-based robust control method was designed to compensate mismatched disturbances and uncertainties in a missile system in [19]. For a nonlinear system in a strict-feedback form with input quantization, actuator faults, and external disturbances, a fuzzy adaptive control scheme based on nonlinear disturbance observer was developed to solve the problem of adaptive fault-tolerant tracking control in a one-link manipulator in [20]. For an underactuated robotic system with matched and mismatched disturbances, a sliding mode controller based on high-order disturbance observer was presented to compensate matched and mismatched disturbances in [21]. However, in [18-21], only single-input-single-output (SISO) nonlinear systems are considered. For a multi-input-multioutput (MIMO) nonlinear system subject to mismatched disturbances, an observer-based sliding mode controller was presented for the discretized system and applied to a 2degree-of-freedom (DOF) serial flexible joint manipulator in [22]. Nevertheless, in [22], the control method requires that the continuous nonlinear system be transformed into a discrete nonlinear system. For a class of $n$-DOF nonlinear system with mismatched and matched disturbances, a sliding mode controller composited with disturbance observer was proposed in [23]. It should be noted that, however, the study in [23] is only applicable to a general nonlinear system without coupling; i.e., $n$ controllers are designed for an $n$-DOF nonlinear system, and each DOF is controlled by its own controller. Nevertheless, for a specific robotic system, it is a strongly coupled system, and it is difficult to be decoupled.

Since robotic system is a nonlinear system with strong coupling and may be subject to various disturbances, this paper is to achieve the position tracking of nonlinear MIMO-coupled robotic system subject to both matched and mismatched disturbances. Matched and mismatched disturbances and the derivative of mismatched disturbance in robotic system are defined as the lumped disturbance. A nonlinear disturbance observer is constructed to estimate the lumped disturbance, and an adaptive law is designed to estimate its bound. An exponential sliding mode controller is derived by combining the nonlinear disturbance observer and exponential convergence law. Stability of the robotic system is analyzed via the Lyapunov function method. The effectiveness of the proposed approach is verified by simulations.

The remainder of this paper is organized as follows: the dynamics of robotic system subject to matched and mismatched disturbances is introduced in Section 2; in Section 3 , the adaptive disturbance observer-based exponential sliding mode controller is designed, and stability of the overall system is analyzed; and simulations on a 2 -DOF robot and conclusion are described in Section 4 and Section 5 , respectively.

\section{Dynamics of Robotic System Subject to Matched and Mismatched Disturbances}

The dynamics of $n$-DOF nonlinear MIMO coupled robotic system without disturbance is considered as follows [24]:

$$
\mathbf{M}(\mathbf{q}) \ddot{\mathbf{q}}+\mathbf{C}(\mathbf{q}, \dot{\mathbf{q}}) \dot{\mathbf{q}}+\mathbf{G}(\mathbf{q})=\tau,
$$

where $\mathbf{q} \in \mathbf{R}^{n}, \dot{\mathbf{q}} \in \mathbf{R}^{n}$, and $\ddot{\mathbf{q}} \in \mathbf{R}^{n}$ are joint angular position, joint angular velocity, and joint angular acceleration, respectively. $\mathbf{M}(\mathbf{q}) \in \mathbf{R}^{n \times n}$ corresponds to symmetric positive definite inertia matrix, $\mathbf{C}(\mathbf{q}, \dot{\mathbf{q}}) \in \mathbf{R}^{n \times n}$ is Coriolis and centrifugal term, $\mathbf{G}(\mathbf{q}) \in \mathbf{R}^{n}$ represents gravity term, and $\tau \in \mathbf{R}^{n}$ denotes control input.

In practice, robotic system is not only subject to matched disturbance but also mismatched disturbance. Therefore, when considering both mismatched and matched disturbances, (1) can be extended to the following state space form:

$$
\left\{\begin{array}{l}
\dot{\mathbf{x}}_{1}=\mathbf{x}_{2}+\mathbf{d}_{1}, \\
\dot{\mathbf{x}}_{2}=-\mathbf{M}^{-1}\left(\mathbf{x}_{1}\right)\left(\mathbf{C}\left(\mathbf{x}_{1}, \mathbf{x}_{2}\right) \mathbf{x}_{2}+\mathbf{G}\left(\mathbf{x}_{1}\right)\right)+\mathbf{M}^{-1}\left(\mathbf{x}_{1}\right) \boldsymbol{\tau}+\mathbf{d}_{2},
\end{array}\right.
$$

where the state vectors $\mathbf{x}_{1} \in \mathbf{R}^{n}$ and $\mathbf{x}_{2} \in \mathbf{R}^{n}$ denote joint angular position and angular velocity of the robotic system, respectively. $\mathbf{d}_{1} \in \mathbf{R}^{n}$ represents mismatched disturbance; i.e., the disturbance $\mathbf{d}_{1}$ is not in the same channel with control input $\tau$ and the disturbance $\mathbf{d}_{1}$ can directly affect the states of the robotic system. In practice, external environmental disturbances, internal parameter uncertainties, and frictions can be considered as mismatched disturbances [9]. $\mathbf{d}_{2} \in \mathbf{R}^{n}$ denotes matched disturbance; i.e., both the disturbance $\mathbf{d}_{2}$ and control input $\tau$ are in the same equation (i.e., the same channel). In practice, the unknown disturbances and the ones with known harmonic frequencies can be considered as matched disturbances [8].

Now, define the lumped disturbance $\mathbf{D}$ in the robotic system:

$$
\mathbf{D}=\mathbf{d}_{1}+\dot{\mathbf{d}}_{1}+\mathbf{d}_{2}
$$

Since disturbance and the derivative of mismatched disturbance (changing rate) are always bounded in practical application $[25,26]$, then

$$
\|\mathbf{D}\| \leq \gamma\|\mathbf{\alpha}\|,
$$

where $\gamma$ is the bound of the lumped disturbance, which is an unknown constant. Besides, $\alpha \in \mathbf{R}^{n}$ will be designed latter. Here, $\|$.$\| refers to the Euclidean norm for the vector, i.e.,$ 
$\|\alpha\|=\sqrt{\alpha_{1}^{2}+\alpha_{2}^{2}+\cdots+\alpha_{n}^{2}}$, where $\alpha_{1}, \alpha_{2}, \ldots, \alpha_{n}$ are the elements of the vector $\alpha$.

Suppose $\mathbf{x}_{d 1}=\mathbf{q}_{d} \in \mathbf{R}^{n}$ is the desired joint angular position trajectory of the robotic system, then we can get the position tracking error as follows:

$$
\mathbf{e}=\mathbf{x}_{1}-\mathbf{x}_{d 1} .
$$

Differentiating (5) and substituting (2) into it, we can obtain the following:

$$
\dot{\mathbf{e}}=\dot{\mathbf{x}}_{1}-\dot{\mathbf{x}}_{d 1} .
$$

Again, differentiating (6) and then substituting (2) into it gives us

$$
\begin{aligned}
\ddot{\mathbf{e}}=\dot{\mathbf{x}}_{2}+\dot{\mathbf{d}}_{1}-\ddot{\mathbf{x}}_{d 1}= & -\mathbf{M}^{-1}\left(\mathbf{x}_{1}\right)\left(\mathbf{C}\left(\mathbf{x}_{1}, \mathbf{x}_{2}\right) \mathbf{x}_{2}+\mathbf{G}\left(\mathbf{x}_{1}\right)\right) \\
& +\mathbf{M}^{-1}\left(\mathbf{x}_{1}\right) \boldsymbol{\tau}+\mathbf{d}_{2}+\dot{\mathbf{d}}_{1}-\ddot{\mathbf{x}}_{d 1} .
\end{aligned}
$$

\section{Design of the Adaptive Disturbance Observer- Based Exponential Sliding Mode Controller}

For the robotic system subject to matched and mismatched disturbances (2), an adaptive disturbance observer-based exponential sliding mode controller is presented to achieve the position tracking performance, as shown in Figure 1. A nonlinear disturbance observer is proposed to estimate the lumped disturbance $\mathbf{D}$ and obtain its estimation $\widehat{\mathbf{D}}$. The bound of the lumped disturbance is estimated by the adaptive law $\hat{\gamma}$ based on the auxiliary variable $\alpha$ of the nonlinear disturbance observer. Then, an exponential sliding mode controller $\tau$ is derived to control the robotic system so that the lumped disturbance is attenuated, and at the same time, the desired joint angular position trajectory $\mathbf{q}_{d}$ can be tracked by the real joint position trajectory $\mathbf{q}$.

3.1. Design of the Nonlinear Disturbance Observer. Since the matched disturbance, mismatched disturbance, and the derivative of mismatched disturbance are defined as the lumped disturbance in robotic system, a nonlinear disturbance observer is constructed to estimate the lumped disturbance, and an adaptive law is proposed to estimate its bound.

For the robotic system subject to matched and mismatched disturbances (2), the nonlinear disturbance observer is proposed as follows:

$$
\begin{aligned}
& \dot{\boldsymbol{\beta}}=-\mathbf{M}^{-1}\left(\mathbf{x}_{1}\right)\left(\mathbf{C}\left(\mathbf{x}_{1}, \mathbf{x}_{2}\right) \mathbf{x}_{2}+\mathbf{G}\left(\mathbf{x}_{1}\right)\right) \\
&+\mathbf{M}^{-1}\left(\mathbf{x}_{1}\right) \boldsymbol{\tau}+(\delta+\widehat{\gamma}) \boldsymbol{\alpha}+\mathbf{x}_{2}-\dot{\mathbf{x}}_{d 1}-\ddot{\mathbf{x}}_{d 1}, \\
& \boldsymbol{\alpha}=\mathbf{e}+\dot{\mathbf{e}}-\boldsymbol{\beta}, \\
& \widehat{\mathbf{D}}=(\delta+\widehat{\gamma}) \boldsymbol{\alpha},
\end{aligned}
$$

where $\alpha \in \mathbf{R}^{n}$ is an auxiliary variable, $\beta \in \mathbf{R}^{n}$ is the state vector of the disturbance observer, $\delta>0$ is a constant, and $\widehat{D}$ is the estimation of the lumped disturbance $\mathbf{D}$.
Although the lumped disturbance is always bounded, the bound is usually unknown. Hence, the adaptive law is designed to estimate its bound as follows:

$$
\dot{\widehat{\gamma}}=\varepsilon \boldsymbol{\alpha}^{T} \boldsymbol{\alpha}
$$

where $\hat{\gamma}$ is the estimation for the unknown bound $\gamma$ of $\mathbf{D}$ and $\varepsilon>0$ is a constant.

Differentiating both sides of (9) and substituting (3) and (6)-(8) into it, we have

$$
\begin{aligned}
\dot{\boldsymbol{\alpha}}= & \mathbf{x}_{2}+\mathbf{d}_{1}-\dot{\mathbf{x}}_{d 1}-\mathbf{M}^{-1}\left(\mathbf{x}_{1}\right)\left(\mathbf{C}\left(\mathbf{x}_{1}, \mathbf{x}_{2}\right) \mathbf{x}_{2}+\mathbf{G}\left(\mathbf{x}_{1}\right)\right) \\
& +\mathbf{M}^{-1}\left(\mathbf{x}_{1}\right) \boldsymbol{\tau}+\mathbf{d}_{2}+\dot{\mathbf{d}}_{1}-\ddot{\mathbf{x}}_{d 1}+\mathbf{M}^{-1}\left(\mathbf{x}_{1}\right) \\
& \cdot\left(\left(\mathbf{C}\left(\mathbf{x}_{1}, \mathbf{x}_{2}\right) \mathbf{x}_{2}+\mathbf{G}\left(\mathbf{x}_{1}\right)\right)\right. \\
& -\mathbf{M}^{-1}\left(\mathbf{x}_{1}\right) \boldsymbol{\tau}-(\delta+\widehat{\gamma}) \boldsymbol{\alpha}-\mathbf{x}_{2}+\dot{\mathbf{x}}_{d 1}+\ddot{\mathbf{x}}_{d 1} \\
= & -(\delta+\widehat{\gamma}) \boldsymbol{\alpha}+\mathbf{D} .
\end{aligned}
$$

According to (10), (12) can be rewritten as

$$
\dot{\boldsymbol{\alpha}}=\mathrm{D}-\widehat{\mathbf{D}} \text {. }
$$

3.2. Design of the Exponential Sliding Mode Controller. Next, the disturbance observer-based exponential sliding mode controller is derived for (2). The sliding mode surface is defined as

$$
\mathbf{s}=\dot{\mathbf{e}}+\mathbf{e}+h \operatorname{sgn}(\mathbf{e}),
$$

where $h>0$ is a constant. sgn is the signum function.

When the position tracking error e of robotic system is in the sliding mode, $\mathbf{s}=0$. Therefore, (14) can be described as

$$
\dot{\mathbf{e}}=-\mathbf{e}-h \operatorname{sgn}(\mathbf{e}) \text {. }
$$

Differentiating (14) along time and substituting (2), (3), (6), and (7) into it, we get

$$
\begin{aligned}
\dot{\mathbf{s}}= & \ddot{\mathbf{e}}+\dot{\mathbf{e}} \\
= & -\mathbf{M}^{-1}\left(\mathbf{x}_{1}\right)\left(\mathbf{C}\left(\mathbf{x}_{1}, \mathbf{x}_{2}\right) \mathbf{x}_{2}+\mathbf{G}\left(\mathbf{x}_{1}\right)\right)+\mathbf{M}^{-1}\left(\mathbf{x}_{1}\right) \boldsymbol{\tau}+\mathbf{d}_{2} \\
& +\dot{\mathbf{d}}_{1}-\ddot{\mathbf{x}}_{d 1}+\mathbf{x}_{2}+\mathbf{d}_{1}-\dot{\mathbf{x}}_{d 1} \\
= & -\mathbf{M}^{-1}\left(\mathbf{x}_{1}\right)\left(\mathbf{C}\left(\mathbf{x}_{1}, \mathbf{x}_{2}\right) \mathbf{x}_{2}+\mathbf{G}\left(\mathbf{x}_{1}\right)\right)+\mathbf{M}^{-1}\left(\mathbf{x}_{1}\right) \tau \\
& -\ddot{\mathbf{x}}_{d 1}+\mathbf{x}_{2}-\dot{\mathbf{x}}_{d 1}+\mathbf{D} .
\end{aligned}
$$

We consider the exponential convergence law in sliding mode control, which is shown as follows:

$$
\dot{\mathbf{s}}=-c \mathbf{s}-K \operatorname{sgn}(\mathbf{s}),
$$

where $K>0$ is a constant switching gain and $c>0$ is a constant.

According to (16) and exponential convergence law (17), the exponential sliding mode controller can be obtained as

$$
\begin{aligned}
\boldsymbol{\tau} & =-\mathbf{M}\left(\mathbf{x}_{1}\right)\left[-\mathbf{M}^{-1}\left(\mathbf{x}_{1}\right)\left(\mathbf{C}\left(\mathbf{x}_{1}, \mathbf{x}_{2}\right) \mathbf{x}_{2}\right.\right. \\
& \left.\left.+\mathbf{G}\left(\mathbf{x}_{1}\right)\right)-\ddot{\mathbf{x}}_{d 1}+\mathbf{x}_{2}-\dot{\mathbf{x}}_{d 1}+\widehat{\mathbf{D}}+c \boldsymbol{\sigma}+K \operatorname{sgn}(\boldsymbol{\sigma})\right],
\end{aligned}
$$

where $\sigma=\mathbf{s}-\alpha$. 
q, $\dot{\mathrm{q}}$

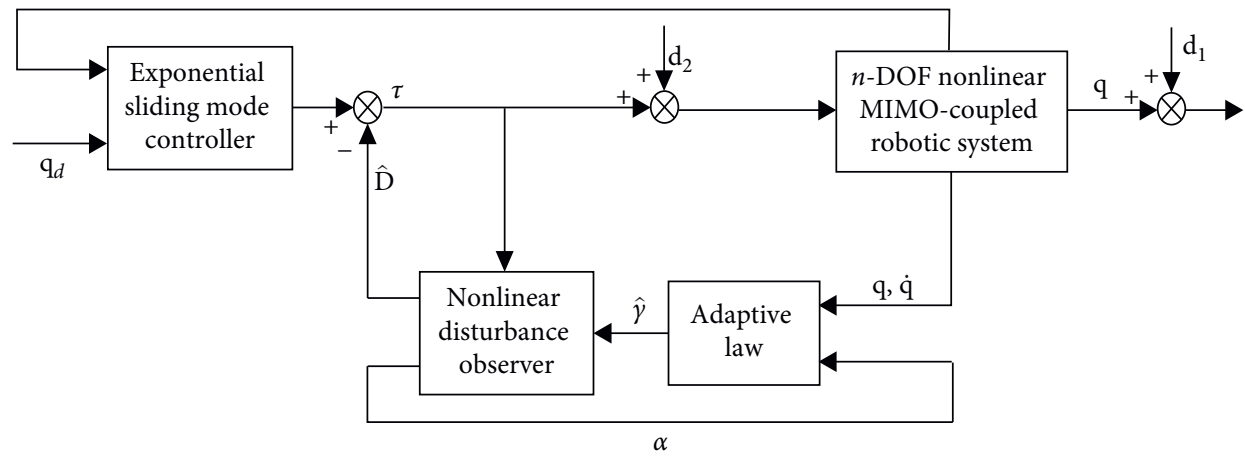

Figure 1: Block diagram of the controller for robotic system subject to matched and mismatched disturbances.

With the sliding mode controller (18) which combines the nonlinear disturbance observer (8)-(10) and the exponential convergence law (17), the matched and mismatched disturbances can be estimated and the position tracking of the robot can be achieved.

Remark 1 . The signum function sgn is utilized in the sliding mode (14) and the exponential sliding mode controller (18). Since sgn is noncontinuous, switching phenomenon will occur in the control process, which can cause chattering. Hence, sgn can be replaced by the saturation function sat [27], which implies that the normal sliding mode control is adopted outside the boundary layer and the continuous state-feedback control is used inside the boundary layer. In this way, the chatting can be avoided or weaken. The saturation function sat is expressed as follows:

$$
\operatorname{sat}\left(\frac{s_{i}}{\Delta}\right)= \begin{cases}1, & s_{i}>\Delta, \\ \frac{s_{i}}{\Delta}, & \left|s_{i}\right| \leq \Delta, \\ -1, & s_{i}<-\Delta,\end{cases}
$$

where $\Delta$ is the thickness of the boundary layer and $\Delta>0$ is a constant, $i=1,2, \ldots, n$.

\subsection{Analysis of the Stability and Tracking Performance of the Robotic System}

Theorem 1. Consider the nonlinear MIMO coupled robotic system (2) is subject to matched and mismatched disturbances. If it is controlled by the exponential sliding mode controller (18) using the nonlinear disturbance observer (8)-(10) and the adaptive law (11), then the following results can be obtained:

(1) The error of the nonlinear disturbance observer (8)-(10) asymptotically converges to zero, i.e., $\lim _{t \longrightarrow \infty}(\mathbf{D}-\widehat{\mathbf{D}})$ $=0$.
(2) The position tracking error e of the robotic system (2) can reach the sliding mode surface $\mathbf{s}=0$.

(3) The position tracking error e asymptotically converges to zero.

Proof. Define a Lyapunov function candidate as follows:

$$
V_{1}=\frac{1}{2} \boldsymbol{\alpha}^{T} \boldsymbol{\alpha}+\frac{1}{2 \varepsilon}(\gamma-\widehat{\gamma})^{2} .
$$

According to (11) and (12), the derivative of (20) can be obtained as follows:

$$
\begin{aligned}
\dot{V}_{1} & =\boldsymbol{a}^{\mathrm{T}} \dot{\boldsymbol{\alpha}}-\frac{\dot{\hat{\gamma}}}{\varepsilon}(\gamma-\widehat{\gamma}) \\
& =\boldsymbol{\alpha}^{T}[-(\delta+\widehat{\gamma}) \boldsymbol{\alpha}+\mathbf{D}]-\boldsymbol{\alpha}^{T} \boldsymbol{\alpha}(\gamma-\widehat{\gamma}) \\
& =-(\delta+\widehat{\gamma})\|\boldsymbol{\alpha}\|^{2}+\boldsymbol{\alpha}^{T} \mathbf{D}-\gamma\|\boldsymbol{\alpha}\|^{2}+\widehat{\gamma}\|\boldsymbol{\alpha}\|^{2} .
\end{aligned}
$$

Substituting (4) into (21), we get

$$
\begin{aligned}
\dot{V}_{1} & =-\delta\|\boldsymbol{\alpha}\|^{2}+\boldsymbol{\alpha}^{T} \mathbf{D}-\gamma\|\boldsymbol{\alpha}\|^{2} \\
& \leq-\delta\|\boldsymbol{\alpha}\|^{2}+\left\|\boldsymbol{\alpha}^{T}\right\|\|\mathbf{D}\|-\gamma\|\boldsymbol{\alpha}\|^{2} \\
& =-\delta\|\boldsymbol{\alpha}\|^{2} \leq 0 .
\end{aligned}
$$

Since $\dot{V}_{1} \leq 0$, it implies $\lim _{t \rightarrow \infty} \alpha=0$. According to Barbalat's lemma [28], we can obtain that $\lim _{t \rightarrow \infty} \dot{\alpha}=0$, and then, $\lim _{t \rightarrow \infty}(\mathbf{D}-\widehat{\mathbf{D}})=0$. Thus, the observer error $(\mathbf{D}-$ $\widehat{\mathbf{D}})$ of the disturbance observer asymptotically converges to zero. This concludes the proof of 1 .

Consider another Lyapunov function candidate as follows:

$$
V_{2}=\frac{1}{2} \boldsymbol{\sigma}^{T} \boldsymbol{\sigma}
$$

Differentiating (23) and substituting the derivative of $\sigma=\mathbf{s}-\alpha,(13)$ and (16) into it, we obtain 


$$
\begin{aligned}
\dot{V}_{2}= & \boldsymbol{\sigma}^{T} \dot{\boldsymbol{\sigma}} \\
= & \boldsymbol{\sigma}^{T}(\dot{\boldsymbol{s}}-\dot{\boldsymbol{\alpha}}) \\
= & \boldsymbol{\sigma}^{T}\left[-\mathbf{M}^{-1}\left(\mathbf{x}_{1}\right)\left(\mathbf{C}\left(\mathbf{x}_{1}, \mathbf{x}_{2}\right) \mathbf{x}_{2}+\mathbf{G}\left(\mathbf{x}_{1}\right)\right)+\mathbf{M}^{-1}\left(\mathbf{x}_{1}\right) \boldsymbol{\tau}\right. \\
& \left.-\ddot{x}_{d 1}+\mathbf{x}_{2}-\dot{x}_{d 1}+\mathbf{D}-(\mathbf{D}-\widehat{\mathbf{D}})\right] \\
= & \boldsymbol{\sigma}^{T}\left[-\mathbf{M}^{-1}\left(\mathbf{x}_{1}\right)\left(\mathbf{C}\left(\mathbf{x}_{1}, \mathbf{x}_{2}\right) \mathbf{x}_{2}+\mathbf{G}\left(\mathbf{x}_{1}\right)\right)+\mathbf{M}^{-1}\left(\mathbf{x}_{1}\right) \boldsymbol{\tau}\right. \\
& \left.-\ddot{x}_{d 1}+\mathbf{x}_{2}-\dot{x}_{d 1}+\widehat{\mathbf{D}}\right] .
\end{aligned}
$$

Substituting the exponential sliding mode controller (18) into (24), it can be obtained that

$$
\begin{aligned}
\dot{V}_{2}= & \boldsymbol{\sigma}^{T}\left[-\mathbf{M}^{-1}\left(\mathbf{x}_{1}\right)\left(\mathbf{C}\left(\mathbf{x}_{1}, \mathbf{x}_{2}\right) \mathbf{x}_{2}+\mathbf{G}\left(\mathbf{x}_{1}\right)\right)+\mathbf{M}^{-1}\left(\mathbf{x}_{1}\right)\right. \\
& \cdot\left(-\mathbf{M}\left(\mathbf{x}_{1}\right)\left(-\mathbf{M}^{-1}\left(\mathbf{x}_{1}\right)\left(\mathbf{C}\left(\mathbf{x}_{1}, \mathbf{x}_{2}\right) \mathbf{x}_{2}+\mathbf{G}\left(\mathbf{x}_{1}\right)\right)-\ddot{x}_{d 1}+\mathbf{x}_{2}\right.\right. \\
& \left.\left.\left.-\dot{x}_{d 1}+\widehat{\mathbf{D}}+c \boldsymbol{\sigma}+K \operatorname{sgn}(\boldsymbol{\sigma})\right)\right)-\ddot{x}_{d 1}+\mathbf{x}_{2}-\dot{x}_{d 1}+\widehat{\mathbf{D}}\right] \\
= & -c \boldsymbol{\sigma}^{T} \boldsymbol{\sigma}-\boldsymbol{\sigma}^{T} K \operatorname{sgn}(\boldsymbol{\sigma}) \\
= & -c\|\boldsymbol{\sigma}\|^{2}-K\|\boldsymbol{\sigma}\| \leq 0 .
\end{aligned}
$$

Therefore, $\sigma^{T} \dot{\sigma} \leq 0$, which implies that $\lim _{t \rightarrow \infty} \sigma=0$, i.e., $\lim _{t \rightarrow \infty}(s-\alpha)=0$. As aforementioned below (22), we have already obtained $\lim _{t \rightarrow \infty} \alpha=0$. Therefore, $\lim _{t \rightarrow \infty} s=0$ is ensured. Thus, according to (14), the position tracking error e can research the sliding mode surface $\mathbf{s}=0$. This concludes the proof of 2 .

Consider the third Lyapunov function candidate as follows:

$$
V_{3}=\frac{1}{2} \mathbf{e}^{T} \mathbf{e} .
$$

Differentiating (26) and substituting (15) into it, we have

$$
\begin{aligned}
\dot{V}_{3} & =\mathbf{e}^{T} \dot{e} \\
& =\mathbf{e}^{T}(-\mathbf{e}-h \operatorname{sgn}(\mathbf{e})) \\
& =-\mathbf{e}^{T} \mathbf{e}-\mathbf{e}^{T} h \operatorname{sgn}(\mathbf{e}) \\
& =-\|\mathbf{e}\|^{2}-h\|\mathbf{e}\| \leq 0 .
\end{aligned}
$$

Hence, $\lim _{t \rightarrow \infty} \mathbf{e}=0$, i.e., the position tracking error $\mathbf{e}$ asymptotically converges to zero. This concludes the proof of 3.

\section{Simulation Studies}

Simulations are conducted on a 2-DOF robot to verify the effectiveness of the proposed controller by using Matlab/ Simulink. The dynamic equation of the 2-DOF robot subject to matched and mismatched disturbances can be written as (2), where
TABle 1: Parameters of the robot.

\begin{tabular}{lc}
\hline Parameter & Value $\left(10^{-3}\right)$ \\
\hline$a_{1}$ & $6.11 \pm 0.9$ \\
$a_{2}$ & $-2.89 \pm 0.43$ \\
$a_{3}$ & $-4.24 \pm 1.01$ \\
$a_{4}$ & $3.01 \pm 0.52$ \\
$a_{5}$ & $2.05 \pm 0.15$ \\
$a_{6}$ & $1.92 \pm 0.23$ \\
$a_{7}$ & $1.60 \pm 0.05$ \\
$a_{8}$ & $-8.32 \pm 2.78$ \\
\hline
\end{tabular}

$$
\begin{aligned}
\mathbf{x}_{1}= & {\left[\begin{array}{ll}
q_{1} & q_{2}
\end{array}\right]^{T}, } \\
\mathbf{x}_{2}= & {\left[\begin{array}{ll}
\dot{q}_{1} & \dot{q}_{2}
\end{array}\right]^{T}, } \\
\mathbf{M}\left(\mathbf{x}_{1}\right)= & {\left[\begin{array}{ll}
m_{11} & m_{12} \\
m_{21} & m_{22}
\end{array}\right], } \\
\mathbf{V}= & \mathbf{C}\left(\mathbf{x}_{1}, \mathbf{x}_{2}\right) \mathbf{x}_{2}+\mathbf{G}\left(\mathbf{x}_{1}\right) \\
= & {\left[\begin{array}{ll}
v_{1} & v_{2}
\end{array}\right]^{T}, } \\
m_{11}= & a_{1}+a_{2} \cos \left(2 q_{2}\right)+a_{3} \sin \left(2 q_{2}\right) \\
& +a_{4} \cos \left(q_{2}\right)+a_{5} \sin \left(q_{2}\right), \\
m_{12}= & m_{21}=0, \\
m_{22}= & a_{6}, \\
v_{1}= & -2 a_{2} \dot{q}_{1} \dot{q}_{2} \sin \left(2 q_{2}\right)+2 a_{3} \dot{q}_{1} \dot{q}_{2} \cos \left(2 q_{2}\right) \\
& +a_{4} \dot{q}_{1} \dot{q}_{2} \cos \left(q_{2}\right)-a_{5} \dot{q}_{1} \dot{q}_{2} \sin \left(q_{2}\right), \\
v_{3}= & 2 a_{2} \dot{q}_{1}^{2} \cos \left(q_{2}\right) \sin \left(q_{2}\right)-a_{3} \dot{q}_{1}^{2} \cos \left(2 q_{2}\right) \\
& -\frac{1}{2} a_{4} \dot{q}_{1}^{2} \cos \left(q_{2}\right)+\frac{1}{2} a_{5} \dot{q}_{1}^{2} \sin \left(q_{2}\right)+a_{7} \sin \left(q_{2}\right) \\
& +a_{8} \cos \left(q_{2}\right) .
\end{aligned}
$$

The parameters of the robot are shown in Table 1 [29].

In simulations, the proposed controller (18) is compared to the traditional sliding mode controller [30]. The sliding mode surface $\mathbf{s}$ and the traditional sliding mode controller $\tau$ are described as follows:

$$
\begin{aligned}
\mathbf{s}= & {\left[\begin{array}{c}
c_{1} e_{q 1}+\dot{e}_{q 1} \\
c_{2} e_{q 2}+\dot{e}_{q 2}
\end{array}\right], } \\
\boldsymbol{\tau}= & \mathbf{M}\left(\mathbf{x}_{1}\right)\left(\left[\begin{array}{c}
c_{1} \dot{e}_{q 1} \\
c_{2} \dot{e}_{q 2}
\end{array}\right]+\ddot{x}_{d 1}+\phi \operatorname{sgn}(\mathbf{s})+\kappa \mathbf{s}\right) \\
& +\mathbf{V}-\mathbf{M}\left(\mathbf{x}_{1}\right)\left(\bar{f}_{p}-\bar{f}_{m} \operatorname{sgn}(\mathbf{s})\right),
\end{aligned}
$$



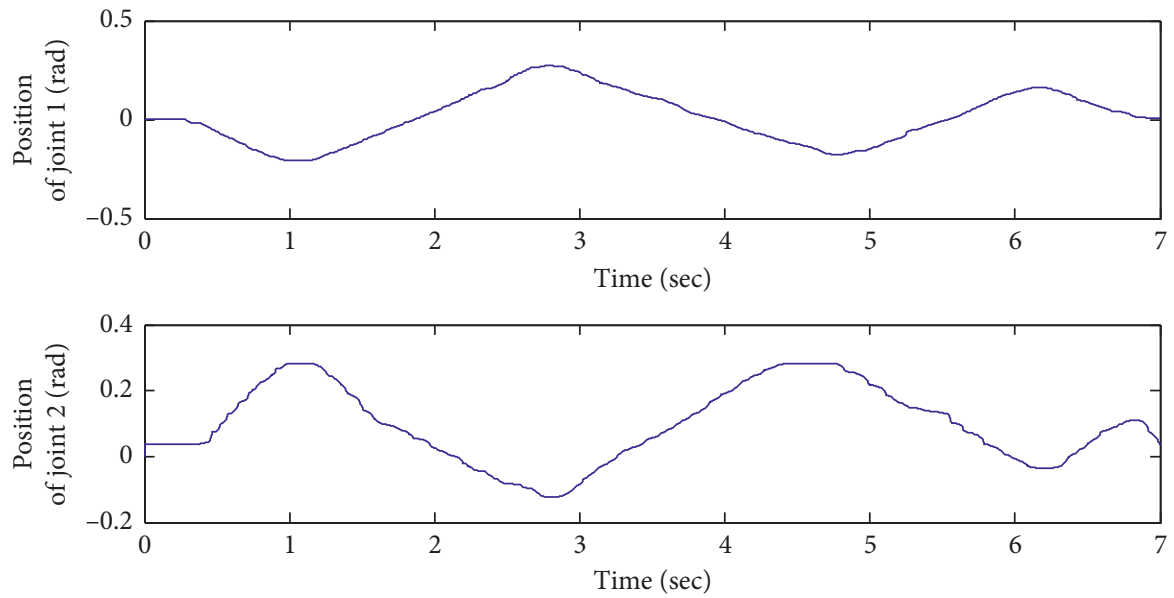

FIgURE 2: Desired joint angular position trajectory.
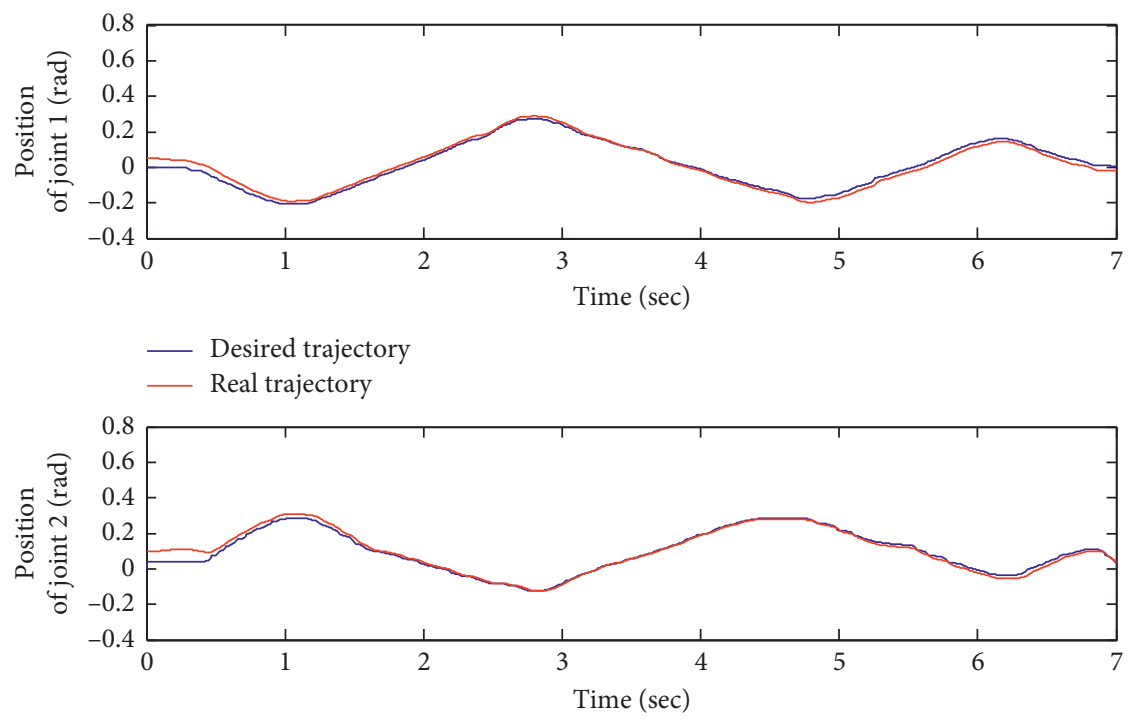

— Desired trajectory

— Real trajectory

(a)

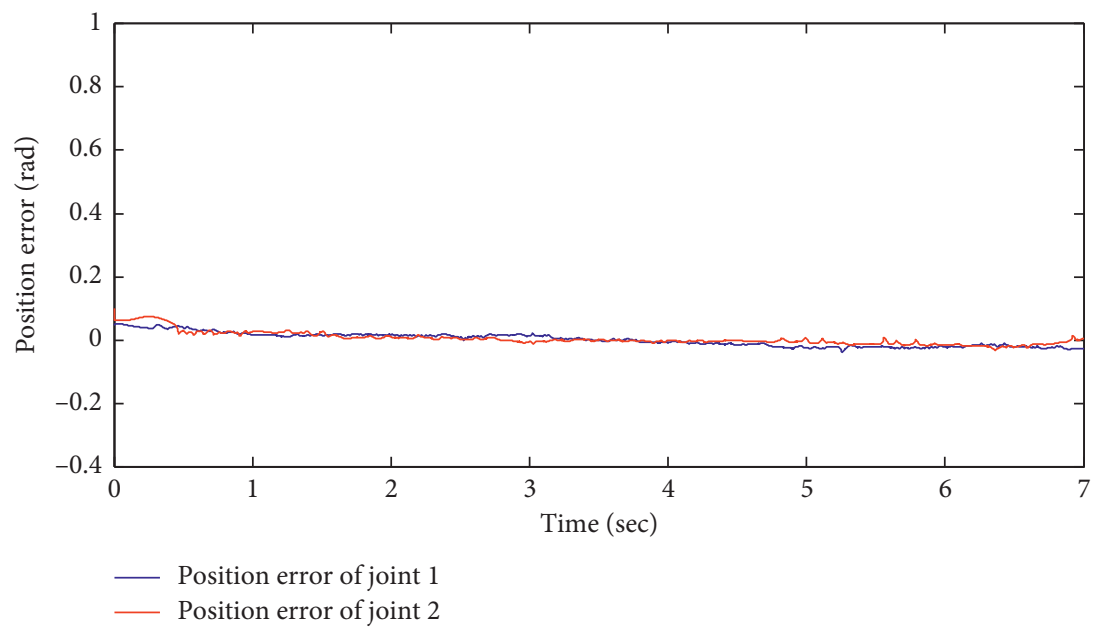

(b)

FIgURE 3: Continued. 


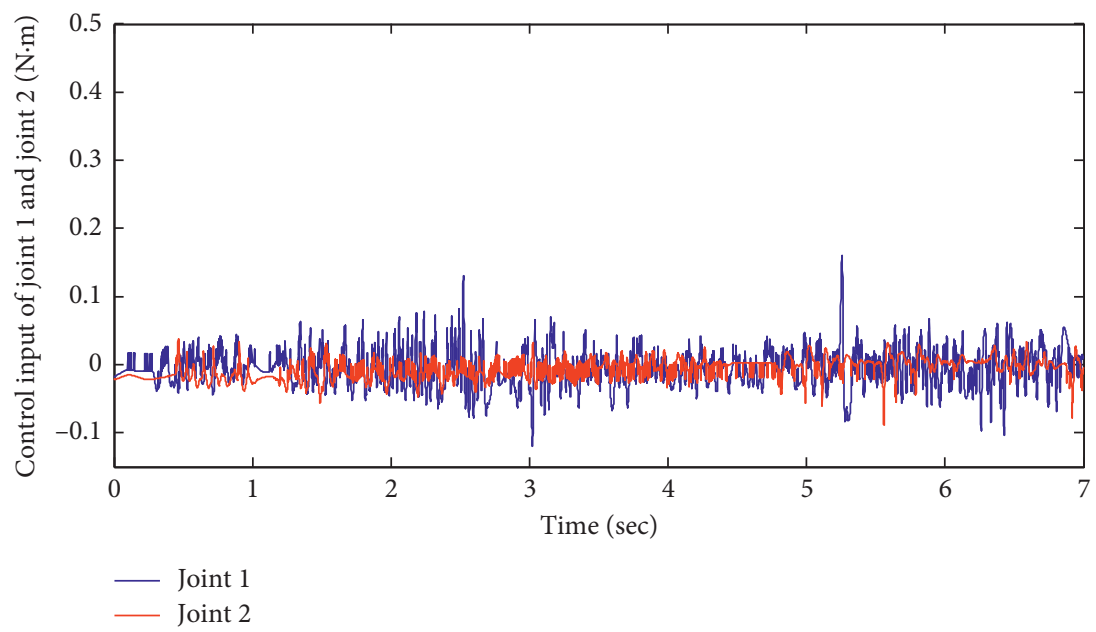

(c)

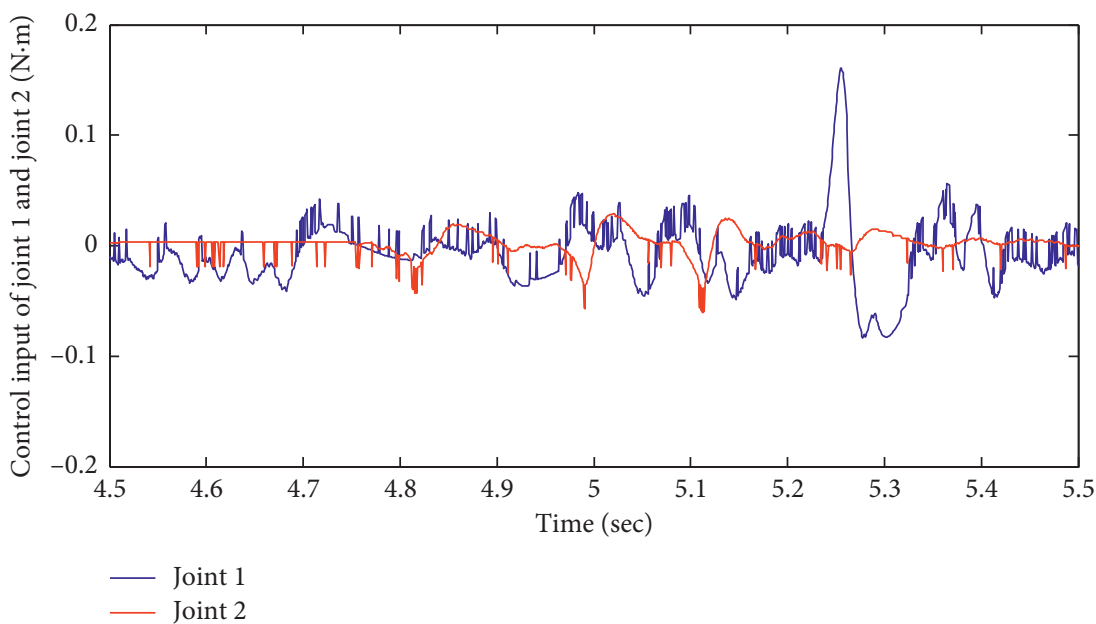

(d)

FIgURE 3: Traditional sliding mode controller: (a) position tracking; (b) position error; (c) control input; (d) enlarged view of the control input.

where $c_{1}>0$ and $c_{2}>0$ are constants; $e_{q 1}$ and $e_{q 2}$ represent position error of joint 1 and joint 2, respectively; $\phi>0$ and $\kappa>0$ are constants; and $\bar{f}_{p}$ and $\bar{f}_{m}$ denote the upper and lower bounds of matched disturbance, respectively.

The traditional sliding mode controller (30) and the proposed controller (18) are compared in the case when the robot is subject to the same matched and mismatched disturbances. The mismatched disturbance and matched disturbance are $\mathbf{d}_{1}=[0.1 \cos (0.5 \mathrm{t}) \quad 0.05 \cos (0.5 \mathrm{t})]^{T}$ and $\mathbf{d}_{2}=\left[0.01 \mathrm{~m}_{11}^{-1} \sin (0.5 \mathrm{t}) \quad 0.01 \mathrm{~m}_{22}^{-1} \cos (0.5 \mathrm{t})\right]^{T}$, respectively. In order to better simulate the practical situation, the desired joint angular position trajectory is chosen in Figure 2. The initial states of the robot are $\mathbf{x}_{1}(0)=\left[\begin{array}{ll}0.05 & 0.1\end{array}\right]^{T}$ and $\mathbf{x}_{2}(0)=\left[\begin{array}{ll}0 & 0\end{array}\right]^{T}$.

When the traditional sliding mode controller is applied, the parameters are $c_{1}=4, c_{2}=4, \kappa=5$, and $\phi=0.5$. The simulation results are shown in Figure 3 .

When the robot is controlled by the proposed controller, the initial values are $\beta_{0}=\left[\begin{array}{ll}0.01 & 0.01\end{array}\right]^{T}$ and $\gamma_{0}=0.01$. The parameters are $\delta=15, \varepsilon=10, h=2, K=0.5$, and $c=40$.
Moreover, the signum functions sgn in (14) and (18) are replaced by the saturation functions sat, and the thickness of the boundary layer is $\Delta=0.01$. The simulation results are shown in Figure 4.

When the robot is controlled by the traditional sliding mode controller, it can be seen from Figures 3(a) and 3(b) that there are obvious position errors between the real trajectory and the desired trajectory and the control inputs have chattering at most of the time in Figure 3(c). On the contrary, when the robot is controlled by the proposed controller, both joint 1 and joint 2 have better tracking performance in Figure 4(a). The position errors approach to zero in Figure 4(b), and the control inputs almost are without chattering in Figure 4(c). Meanwhile, from Figure 4(d), the lumped disturbances of joint 1 and joint 2 are estimated well by the designed disturbance observer.

In order to further quantitatively compare the position tracking performance of those two control approaches, the settling time $t_{s}( \pm 4 \%)$ and mean square error (MSE) $E_{\mathrm{MSE}}$ are calculated in Table 2 . 

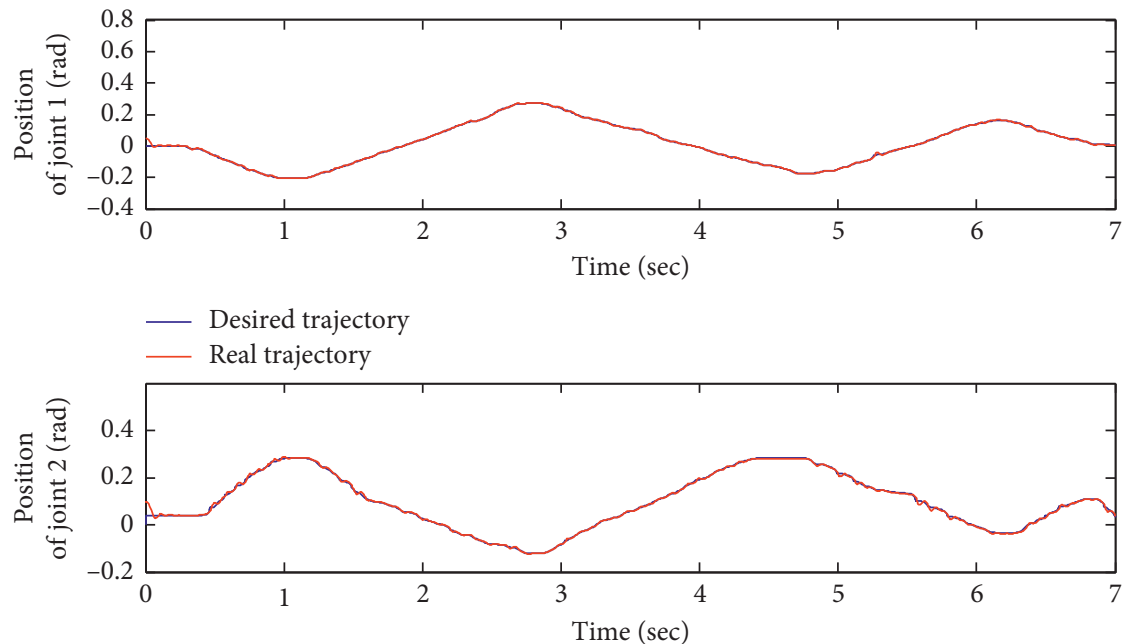

— Desired trajectory

— Real trajectory

(a)

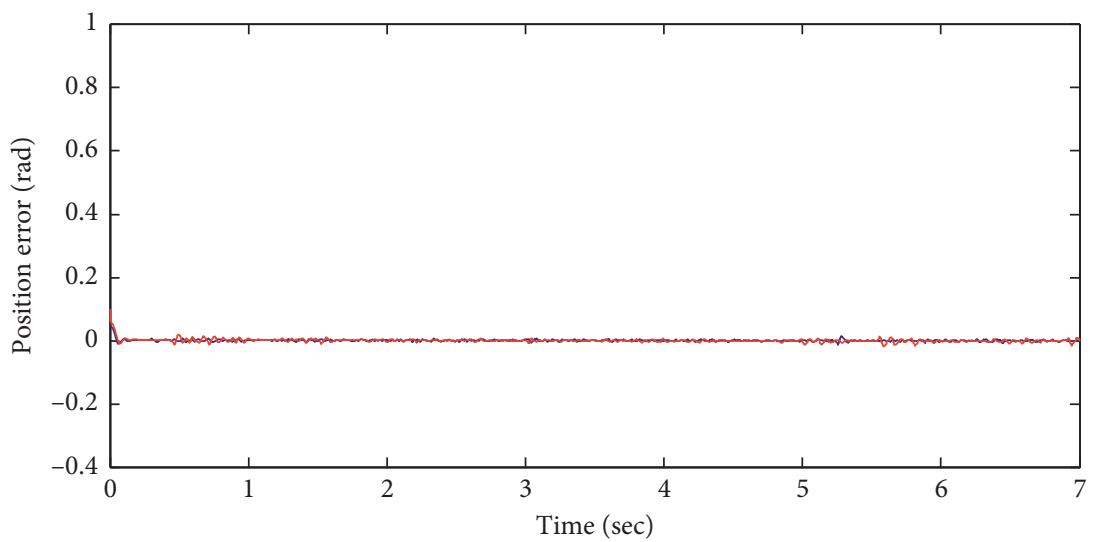

- Position error of joint 1

Position error of joint 2

(b)

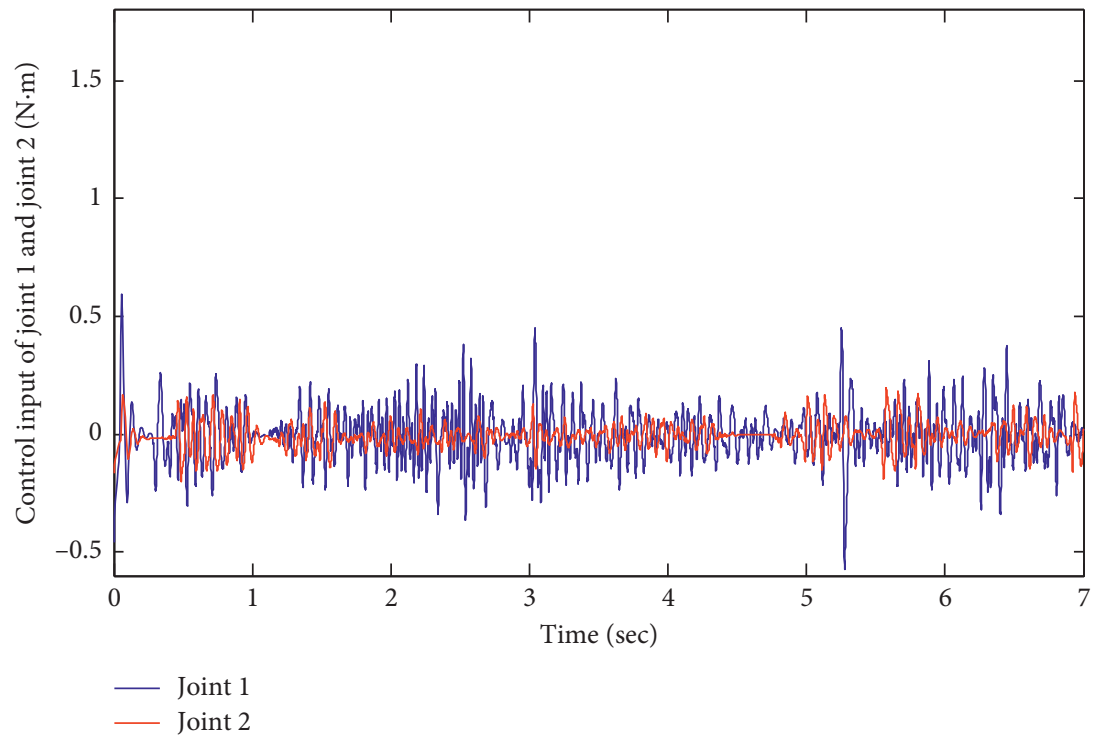

(c)

FIGURE 4: Continued. 


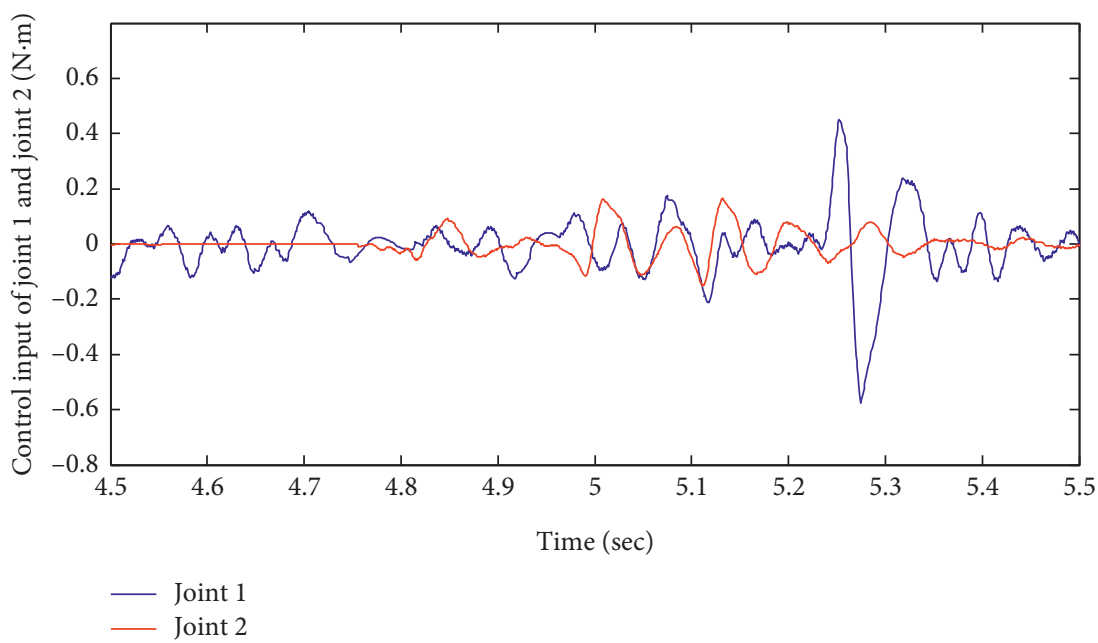

(d)

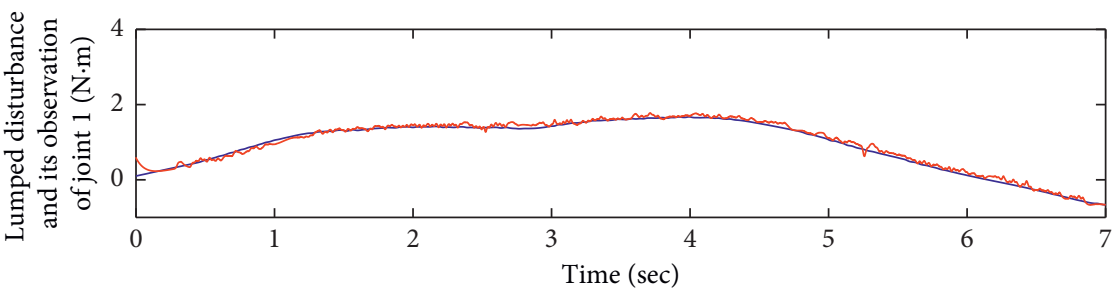

- Real disturbance

— Disturbance observation

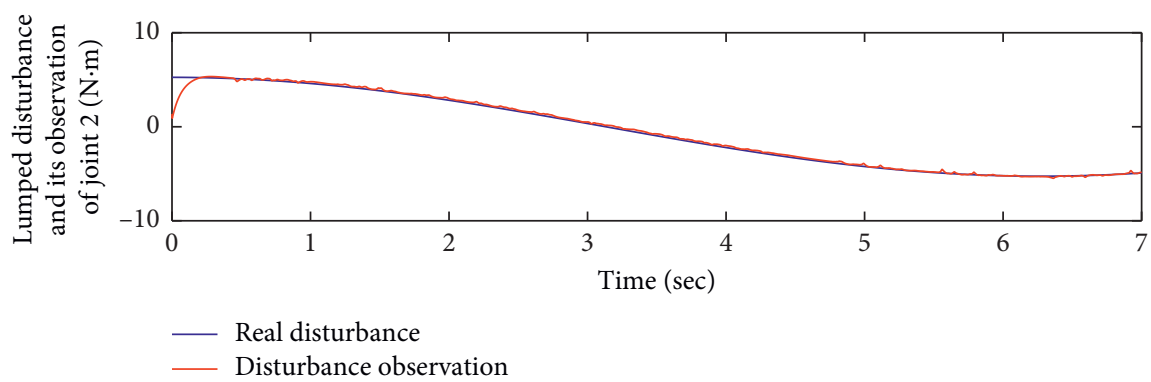

(e)

Figure 4: Proposed adaptive disturbance observer-based exponential sliding mode controller: (a) position tracking; (b) position error; (c) control input; (d) enlarged view of the control input; (e) lumped disturbances and its observation.

When the traditional sliding mode controller is used, as shown in Table 2, the settling time of position tracking of joint 1 and joint 2 is $0.5384 \mathrm{~s}$ and $0.4544 \mathrm{~s}$, respectively, and the MSE of joint 1 and joint 2 is $0.01976 \mathrm{rad}$ and $0.01843 \mathrm{rad}$, respectively. By comparison, when the proposed controller is employed, the settling time of position tracking of joint 1 and joint 2 in robotic system is $0.01774 \mathrm{~s}$ and $0.03014 \mathrm{~s}$, respectively, and the MSE of joint 1 and joint 2 is $0.002778 \mathrm{rad}$ and $0.004691 \mathrm{rad}$, respectively. The settling time is much shorter, and MSE is much smaller.

In general, according to Figures 3 and 4 and Table 2, the position tracking performance of the proposed controller is significantly better than that of the traditional sliding mode controller. The reason for this phenomenon is that the latter
TABLE 2: Quantitative comparison of position tracking performance.

\begin{tabular}{lcccc}
\hline & \multicolumn{2}{c}{$\begin{array}{c}\text { Traditional sliding } \\
\text { mode controller }\end{array}$} & \multicolumn{2}{c}{$\begin{array}{c}\text { Adaptive disturbance } \\
\text { observer-based } \\
\text { exponential sliding } \\
\text { mode controller }\end{array}$} \\
& $t_{s}(\mathrm{~s})$ & $E_{\mathrm{MSE}}(\mathrm{rad})$ & $t_{s}(\mathrm{~s})$ & $E_{\mathrm{MSE}}(\mathrm{rad})$ \\
\hline Joint 1 & 0.5384 & 0.01976 & 0.01774 & 0.002778 \\
Joint 2 & 0.4544 & 0.01843 & 0.03014 & 0.004691 \\
\hline
\end{tabular}

cannot compensate the mismatched disturbance, while the former can attenuate the matched and mismatched disturbances in robotic system. 


\section{Conclusions}

This paper studies the estimation and attenuation of matched and mismatched disturbances for nonlinear MIMO-coupled robotic system. An adaptive disturbance observer-based exponential sliding mode controller is proposed to achieve position tracking. The simulation results show that, with the proposed approach, both matched and mismatched disturbances in robotic system can be effectively depressed while achieving position tracking. Conducting experiments to verify the effectiveness of the proposed approach remains as our future work. Besides, in practice, the disturbance in a robotic system may even be aperiodic, e.g., random disturbance. Thus, control of robotic system with random disturbance in nonlinear MIMO robotic system will also be addressed in our future research.

\section{Data Availability}

The data that supports our manuscript conclusions are some open-access articles that have been properly cited, and the readers can easily obtain these articles to verify the conclusions.

\section{Conflicts of Interest}

The authors declare that they have no conflicts of interest regarding the publication of this paper.

\section{Acknowledgments}

This research was supported by the National Natural Science Foundation of China (Grant nos. 61973257, 61973331, and 61875166) and Sichuan Science and Technology Program (Grant no. 2020YJ0367).

\section{References}

[1] Y. Chen, Z. Li, H. Kong, and F. Ke, "Model predictive tracking control of nonholonomic mobile robots with coupled input constraints and unknown dynamics," IEEE Transactions on Industrial Informatics, vol. 15, no. 6, pp. 3196-3205, 2019.

[2] D. C. Chen, S. A. Li, Q. Wu, and X. Luo, "New disturbance rejection constraint for redundant robot manipulators: an optimization perspective," IEEE Transactions on Industrial Informatics, vol. 16, no. 4, pp. 2221-2232, 2020.

[3] X. Shao, B. Tian, W. Yang, and Z. Wendong, "Estimator-based MLP neuroadaptive dynamic surface containment control with prescribed performance for multiple quadrotors," Aerospace Science and Technology, vol. 97, p. 105620, 2020.

[4] X. Shao, L. Wang, J. Li, and J. Liu, "High-order ESO based output-feedback dynamic surface control for quadrotors under position constraints and uncertainties," Aerospace Science and Technology, vol. 89, pp. 228-298, 2019.

[5] X. Shao, H. Si, and W. Zhang, "Fuzzy wavelet neural control with improved prescribed performance for mems gyroscope subject to input quantization," Fuzzy Sets and Systems, In press.

[6] W.-H. Chen, J. Yang, L. Guo, and S. Li, "Disturbance-observer-based control and related methods-an overview," IEEE Transactions on Industrial Electronics, vol. 63, no. 2, pp. 1083-1095, 2016.
[7] X. Yang, P. Wei, Y. Zhang, X. Liu, and L. Yang, "Disturbance observer based on biologically inspired integral sliding mode control for trajectory tracking of mobile robots," IEEE Access, vol. 7, pp. 48382-48391, 2019.

[8] Z. Sun, Y. Xia, L. Dai, K. Liu, and D. Ma, "Disturbance rejection MPC for tracking of wheeled mobile robot," IEEE/ ASME Transactions on Mechatronics, vol. 22, no. 6, pp. 2576-2587, 2017.

[9] H. Wang, Y. Pan, S. Li, and H. Yu, "Robust sliding mode control for robots driven by compliant actuators," IEEE Transactions on Control Systems Technology, vol. 27, no. 3, pp. 1259-1266, 2019.

[10] W. J. Chen and M. Tomizuka, "Dual-stage iterative learning control for MIMO mismatched system with application to robots with joint elasticity," IEEE Transactions on Control Systems Technology, vol. 22, no. 4, pp. 1350-1361, 2014.

[11] X. Shao, J. Liu, H. Cao, C. Shen, and H. Wang, "Robust dynamic surface trajectory tracking control for a quadrotor UAV via extended state observer," International Journal of Robust and Nonlinear Control, vol. 28, no. 7, pp. 2700-2719, 2018.

[12] X. Liu and D. Liu, "Composite control of nonlinear robotic system with exogenous disturbance," IEEE Access, vol. 7, pp. 19564-19571, 2019.

[13] J. Ferguson, D. Wu, and R. Ortega, "On matched disturbance suppression for Port-Hamiltonian systems," IEEE Control Systems Letters, vol. 4, no. 4, pp. 892-897, 2020.

[14] Z. Li, C.-Y. Su, L. Wang, Z. Chen, and T. Chai, "Nonlinear disturbance observer-based control design for a robotic exoskeleton incorporating fuzzy approximation," IEEE Transactions on Industrial Electronics, vol. 62, no. 9, pp. 5763-5775, 2015.

[15] J. N. Yun and J. B. Su, "Design of a disturbance observer for a two-link manipulator with flexible joints," IEEE Transactions on Control Systems Technology, vol. 22, no. 2, pp. 809-815, 2014.

[16] X. Shao and Y. Shi, "Neural adaptive control for MEMS gyroscope with full-state constraints and quantized input," IEEE Transactions on Industrial Informatics, vol. 16, no. 10, pp. 6444-6454, 2020.

[17] J. Zhang, X. Liu, Y. Xia, Z. Zuo, and Y. Wang, "Disturbance observer-based integral sliding-mode control for systems with mismatched disturbances," IEEE Transactions on Industrial Electronics, vol. 63, no. 11, pp. 7040-7048, 2016.

[18] L. Zhou, L. Cheng, J. She, and Z. Zhang, "Generalized extended state observer-based repetitive control for systems with mismatched disturbances," International Journal of Robust and Nonlinear Control, vol. 29, no. 11, pp. 3777-3792, 2019.

[19] J. Yang, W.-H. Chen, and S. Li, "Non-linear disturbance observer-based robust control for systems with mismatched disturbances/uncertainties," IET Control Theory \& Applications, vol. 5, no. 18, pp. 2053-2062, 2011.

[20] Y. H. Jing and G. H. Yang, "Fuzzy adaptive quantized faulttolerant control of strict-feedback nonlinear systems with mismatched external disturbances," IEEE Transactions on Systems, Man, and Cybernetics: Systems, vol. 50, no. 9, 2020.

[21] J. Huang, S. Ri, T. Fukuda, and Y. Wang, "A disturbance observer based sliding mode control for a class of underactuated robotic system with mismatched uncertainties," IEEE Transactions on Automatic Control, vol. 64, no. 6, pp. 2480-2487, 2019.

[22] D. Ginoya, P. D. Shendge, and S. B. Phadke, "Delta-operatorbased extended disturbance observer and its applications," 
IEEE Transactions on Industrial Electronics, vol. 62, no. 9, pp. 5817-5828, 2015.

[23] M. P. Aghababa, "Sliding-mode control composite with disturbance observer for tracking control of mismatched uncertain nDoF nonlinear systems," IEEE/ASME Transactions on Mechatronics, vol. 23, no. 1, pp. 482-490, 2018.

[24] T. Li, S. Duan, J. Liu, L. Wang, and T. Huang, "A spintronic memristor-based neural network with radial basis function for robotic manipulator control implementation," IEEE Transactions on Systems, Man, and Cybernetics: Systems, vol. 46, no. 4, pp. 582-588, 2016.

[25] W. Liang, S. Huang, S. Chen, and K. K. Tan, "Force estimation and failure detection based on disturbance observer for an ear surgical device," ISA Transactions, vol. 66, pp. 476-484, 2017.

[26] X. Shao, N. Liu, Z. Wang, W. Zhang, and W. Yang, "Neuroadaptive integral robust control of visual quadrotor for tracking a moving object," Mechanical Systems and Signal Processing, vol. 136, p. 106513, 2020.

[27] M. Li and Y. Chen, "Robust adaptive sliding mode control for switched networked control systems with disturbance and faults," IEEE Transactions on Industrial Informatics, vol. 15, no. 1, pp. 193-204, 2019.

[28] J. J. E. Slotine and W. Li, Applied Nonlinear Control, PrenticeHall, Englewood Cliffs, NJ, USA, 1991.

[29] A. Mohammadi, M. Tavakoli, H. J. Marquez, and F. Hashemzadeh, "Nonlinear disturbance observer design for robotic manipulators," Control Engineering Practice, vol. 21, no. 3, pp. 253-267, 2013.

[30] S. Islam and X. P. Liu, "Robust sliding mode control for robot manipulators," IEEE Transactions on Industrial Electronics, vol. 58, no. 6, pp. 2444-2453, 2011. 